\title{
Injuries of the sporting knee
}

The first article in this series drew attention to the evidence that ligament damage is more common than any other type of knee injury pathology. The mean delay from anterior cruciate ligament (ACL) injury to diagnosis at a specialist knee clinic was reported as 22 months. The management of the resulting instability from isolated ligament injuries and from more complex injuries is the subject of this the next article in the series.

The paper has been written by Derek Bickerstaff and Trinath Kakarlapudi from the Northern General Hospital in Sheffield. Derek Bickerstaff has a special interest in complex knee injuries and revision surgery. He is a member of the advisory committee producing national guidelines for the practice of ACL reconstruction and he has close associations with top level teams in several sports. Since his sports surgery fellowship in Adelaide, Australia, he has published and presented prolifically on the subject of knee injuries. His colleague, Trinath Kakarlapudi, is a specialist registrar who has just begun a fellowship in knee surgery in Toronto, Canada.

The article addresses the issues of assessment, treatment, and rehabilitation of the major isolated ligament injuries as well as the common combined instability patterns. It makes the point that treatment is aimed at restoring stability and function rather than preventing long term degenerative change, as there is no evidence that reconstruction reduces the development of arthritis. However, evidence is emerging that ACL reconstruction may reduce the incidence of subsequent meniscal damage and allow retention of their protective effect. ACL reconstruction is now such a popular and common operation world wide that data comparing the long term results of modern methods of reconstruction with the natural history of the deficient knee will soon be available.

For the future, the challenges mentioned are prevention of associated degenerative change and advances in prosthetic structures rather than grafts.

I CORRY

Consultant Orthopaedic Surgeon

Royal Victoria Hospital, Belfast

Consultant Orthopaedic Surgeon

J WEBB

Southmead Hospital, Bristol

\section{Knee instability: isolated and complex}

The past decade has seen several advances in the understanding, evaluation, treatment, and rehabilitation of knee instabilities. Despite these advances, an unstable knee still poses many challenges to the treating clinician because of the complexity of its nature and the demands of the patients who are usually young and active sport enthusiasts. This article presents an overview of the various aspects of knee ligament instabilities.

\section{Stability and instability}

Stability of the knee joint is maintained by the shape of the condyles and menisci in combination with passive supporting structures. These are the four major ligaments, the anterior cruciate ligament (ACL), the posterior cruciate ligament (PCL), the medial collateral ligament (MCL), and the lateral collateral ligament (LCL). Significant contributions are also made by the posteromedial and posterolateral capsular components and the iliotibial tract. The muscles acting over the joint provide secondary dynamic stability.

Instability resulting from ligament injury may result from direct or indirect trauma. The most frequent mechanism is "non-contact" involving cutting, twisting, jumping, and sudden deceleration.

\section{Assessment}

This begins with a detailed history, including a description of the injury. The timing of an effusion (acute haemarthrosis usually occurs within two hours) and hearing or feeling a "pop" (highly suggestive of an ACL injury) are significant events. Chronic instabilities present with mechanical symptoms such as locking, catching, clicking, or giving way, particularly with twisting movements. Age, occupation, lifestyle, level of sporting activity, and past history are all factors considered in subsequent management. Initial physical examination may be difficult because of swelling, pain, or muscle spasm. The specific physical signs are described below. Investigations must include plain radiographs of the knee. These may show fractures, avulsions, osteochondral fragments, or the fluid level of a haemarthrosis.

If a clear diagnosis is made, a specific treatment can be started. If an adequate examination is possible, but diagnosis is inconclusive, an expectant policy of mobilisation, physiotherapy and re-evaluation in about two weeks may be adopted. If adequate examination is not possible because of pain, spasm, etc, the options available are re-evaluation, magnetic resonance imaging (MRI), or examination under anaesthesia and arthroscopy. MRI is particularly useful because of its non-invasive nature, but it is not universally available in the United Kingdom as an emergency investigation.

\section{Medial collateral ligament}

ANATOMY AND FUNCTION

The MCL is attached proximally to the medial femoral condyle and distally to the tibial metaphysis, $4-5 \mathrm{~cm}$ distal to the medial joint line beneath the pes anserinus insertion. Posterior to the MCL is the posterior oblique ligament which is a thickening of the capsule. Immediately deep to 
the MCL is the medial capsular ligament. These constitute the medial ligament complex. In full extension, the posterior oblique ligament and the posteromedial capsule resist valgus stresses. These relax at $20-30^{\circ}$ of flexion, when the MCL becomes the primary restraint. The MCL together with the posterior oblique ligament also resists abnormal internal tibial rotation. Isolated $\mathrm{MCL}$ injuries occur usually as a result of a direct blow to the lateral aspect of the knee in a slightly flexed position. When the deforming force includes a rotational component, associated injuries to the cruciate ligaments can occur.

\section{DIAGNOSIS OF INJURY}

Physical examination includes looking for a localised bruise or swelling or localised tenderness, and application of a gentle valgus force with the patient's knee in $15-20^{\circ}$ of flexion. The degree of medial joint opening compared with the normal knee is a measure of damage to the MCL. A difference of only $5 \mathrm{~mm}$ indicates significant structural damage to the MCL. Excessive opening in full extension indicates combined MCL and posterior oblique ligament damage and should alert the examiner to the strong possibility of an associated ACL or PCL injury. If the knee is stable in full extension, one can safely assume that there is no significant damage to the posterior oblique ligament.

\section{MANAGEMENT OF INJURIES}

Treatment of acute isolated MCL injury is conservative. ${ }^{1}$ Incomplete tears of the MCL (sprains) without significant instability are treated with rest, ice, compression, and elevation (RICE) during the first 48 hours. This is followed by temporary immobilisation and the use of crutches for pain control. Weight bearing as tolerated is encouraged as soon as pain allows. Early mobilisation and physiotherapy allows patients to return to activities within about six weeks.

\section{CHRONIC MCL INSUFFICIENCY}

This is rare in isolation and usually is associated with ACL or PCL injury. Careful examination must differentiate between MCL with or without posteromedial rotary instability. Symptomatic medial instability not improved with conservative treatment usually requires surgery in the form of proximal advancement of the MCL. ${ }^{2}$ Posteromedial rotary instability may require reconstruction of the posterior oblique ligament with free hamstring tendon graft.

\section{Anterior cruciate ligament}

ANATOMY AND FUNCTION

The femoral attachment is on the lateral wall of the intercondylar notch posteriorly. The tibial attachment is on the anterior part of the tibial plateau near the tibial spines. The ACL has an anteromedial band which is tighter in flexion and a posterolateral band which is tighter in extension. ${ }^{3}$ This arrangement allows different portions to be taut throughout the range of motion, allowing the ligament to be functional throughout flexion and extension. It has also been shown to contain proprioceptive nerve endings. ${ }^{4}$

The ACL is the primary restraint to anterior translation of the tibia on the femur and to hyperextension. ${ }^{5}$ It functions as a secondary restraint to varus or valgus angulation at full extension. It also resists internal and external rotation at near full extension.

\section{DIAGNOSIS OF INJURY}

\section{Lachman's test}

This is performed with the knee in $20-30^{\circ}$ of flexion with the femur stabilised. An anterior force is applied to the proximal tibia and the displacement is assessed.
The pivot shift test

This is a dynamic test that shows the subluxation that occurs when the ACL is non-functional. In early flexion, the anterolateral quadrant of the tibia is forced to sublux by internal rotation and valgus. This reduces with a clunk by the posterior pull of the iliotibial tract with further flexion up to $20-40^{\circ}$.

Plain radiographs may show an avulsion of the insertion of the ACL or a Segond fracture, which is a lateral capsular avulsion fracture from the margin of the lateral tibial plateau. MRI has an overall accuracy of about $90 \%$ in assessing the ACL, ${ }^{6}$ although this is not required routinely. MRI also shows "bone bruises" seen in about $60 \%$ of ACL injuries, ${ }^{7}$ the significance and long term sequelae of which have yet to be determined. Instrumented Lachman testing with the KT1000 arthrometer allows documentation of anteroposterior displacement before and after surgery. Examination under anaesthesia and arthroscopy for diagnosis is required only if there is still doubt after clinical examination and MRI scan.

MANAGEMENT OF INJURIES

It is widely accepted that an acute repair is associated with poor results including a higher rerupture rate and arthrofibrosis. ${ }^{8}$ Hence the initial treatment is based on the reduction of pain and swelling and early restoration of normal joint movement. The goal of treatment for ACL deficiency is to prevent reinjury, which may lead to chondral damage, meniscal tear, or laxity of secondary restraints. These secondary injuries are thought to lead to arthritis, although progression to radiologically detectable osteoarthritis appears to be variable. ${ }^{9}$ We are aware of no study in the literature proving that ACL reconstruction to stabilise the knee prevents the development of arthritis.

Once ACL deficiency is diagnosed, the decision between operative and non-operative treatment is based on variables that are unique to each person. Among the factors considered are the patient's age, activity level (recreational/ occupational), the degree of laxity, associated meniscal or ligamentous pathology, ability and willingness to participate in a physiotherapy programme, and future expectations, including the type of sporting activity in which the patient wishes to participate.

Daniel et $a l^{10}$ have shown that the ability of a patient to cope with ACL insufficiency is related to both the amount of instability present and the willingness to modify lifestyle avoiding high risk activities. This prospective outcome study followed up 292 patients for an average of five years. In total, $19 \%$ underwent ACL reconstruction within the first three months, $19 \%$ requested surgery over the next five years, and $62 \%$ were able to function satisfactorily without an ACL. Those who had less than $5 \mathrm{~mm}$ of side to side difference and who participated for 50 hours or less in level 1 or 2 sports had a low risk of needing further surgery. Those with a $7 \mathrm{~mm}$ or greater side to side difference with more than 50 hours of level 1 or 2 sporting activity were in the high risk group.

Activities can be graded depending on the risk to the ACL deficient knee. Low risk (level 3) includes cycling, swimming, stair climbing, and rowing, and medium risk (level 2) includes skiing, tennis, and golf. Although level 2 sports involve pivoting, this is predictable and a patient can usually prepare for it. High risk (level 1) sports include high level skiing, basketball, football, and volleyball where there is considerable risk that the patient can be caught offguard and suffer a twisting injury without time to prepare. Patients with ACL insufficiency are best advised to avoid participation in level 1 sports.

Older people are often more willing to modify their activities, but surgery may be required if the laxity level is 
so great that their activities of daily living are impaired. As patients do not tolerate instability in two major ligaments well, the presence of associated injuries also influences decision making in favour of surgery. Also ACL reconstruction is advisable in cases in which meniscal repair is undertaken, because the failure rate of meniscal repair is too large in the presence of ACL instability. ${ }^{11}$

\section{Conservative management}

Non-operative management of acute ACL tears is likely to be successful in those patients who have no associated injuries, and are willing to give up high demand sports. The rehabilitation programme emphasises proprioceptive training to maximise the dynamic stability. Non-operative management also includes counselling about high risk activities and measures to prevent recurrent injuries.

The role of functional knee bracing remains controversial. ${ }^{12}$ These may provide protection by improving joint position sense and by providing mechanical constraint of joint motion. Some patients report that they can participate in an increased level of sporting activity; however, the use of a brace cannot substitute for lack of quadriceps or hamstring training and cannot ensure protection from further injury.

\section{Surgical management}

Surgical techniques have been described for intra-articular and extra-articular reconstructions of the ACL, using iliotibial band, the semitendinosus and gracilis tendons, the patella tendon, allograft tissue, and various synthetic materials. Currently intra-articular techniques are most commonly used. The surgical technique requires proper placement and tensioning of the graft, avoidance of impingement and stress risers on the implanted tissue, and adequate fixation.

The available graft materials are broadly divided into autografts, allografts, and synthetic grafts. Autogenous grafts are most commonly used in ACL reconstruction. They provide a framework for revascularisation and regeneration of the ligament and, with modern fixation techniques, allow rapid rehabilitation. Allografts heal in a similar fashion but at a slower rate. There is also a risk of disease transmission. They are therefore more widely used when there is no autograft alternative. Synthetic grafts, although theoretically the most attractive, have not proved successful in the long term.

Surgeons differ in their preference for autogenous tissue. The patella tendon graft (B-PT-B) allows more secure bone to bone fixation. Most Surgeons report $80-90 \%$ good or excellent results using autogenous B-PT-B (fig 1). Patellar fracture, tendinitis, anterior knee pain, and increased incidence of infrapatellar contracture have been described with their use. Patellar fracture can usually be avoided by careful technique. Patellar tendinitis is usually short lived, and after one year is generally not a problem. Anterior knee pain, however, appears to be more significant with this graft source than with hamstring reconstruction.

Use of combined semitendinosus and gracilis tendon grafts for reconstruction of ACL has also been well established. Their stiffness characteristics mimic the normal ACL more closely than the stiffer patellar tendon graft. Multiple strands of the hamstring grafts may allow a better opportunity for revascularisation. They offer an alternative in skeletally immature patients (where harvesting patellar graft would jeopardise the tibial apophysis), in women for cosmetic reasons or in patients with extensor mechanism pathology. Hamstring harvest is associated with minimal graft site morbidity. ${ }^{13}$ Both direct and indirect clinical comparisons have shown that B-PT-B grafts and hamstring grafts have similar rates of effectiveness in adults with only

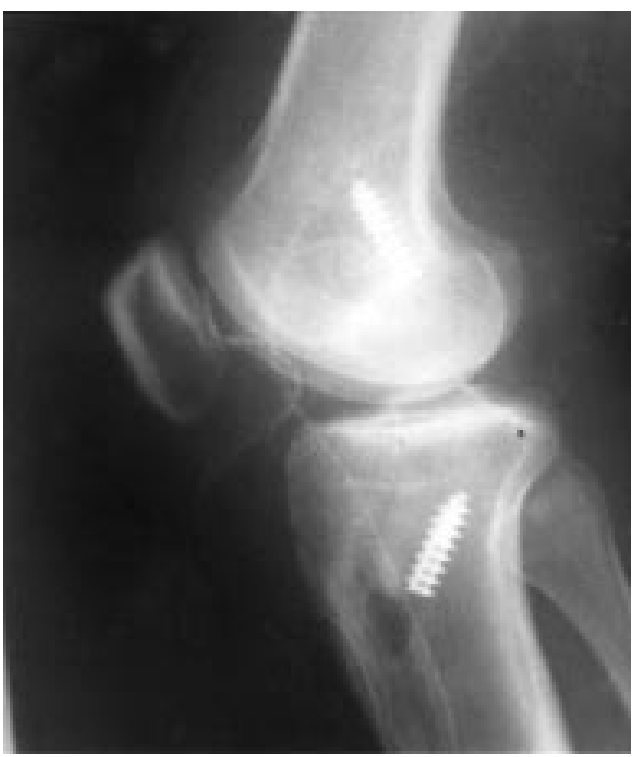

Figure 1 Lateral radiograph of the knee showing two interference screws used to secure a patella tendon graft in reconstruction of the anterior cruciate ligament.

minimal variations in knee stability and muscle strength at an average of three years after implantation. ${ }^{14}$

\section{REHABILITATION}

Postoperative rehabilitation is an important aspect of care of the ACL reconstruction. Shelbourne and $\mathrm{Nitz}^{15}$ have advocated accelerated rehabilitation, with an objective being early and long term maintenance of full knee extension. This protocol was based on the use of patellar tendon graft, although the principles are similar with other types of grafts.

\section{Lateral collateral ligament and posterolateral corner}

ANATOMY AND FUNCTION

The LCL originates on the lateral epicondyle of the femur and is attached distally on the fibular head. It is relatively rarely injured in isolation and is usually injured as part of a complex involving the posterolateral corner (PLC), the PCL, or the ACL. The PLC is a complex anatomical region of the knee consisting of the popliteus tendon, the popliteofibular ligament, the arcuate ligament, and the posterolateral joint capsule. The lateral and posterolateral corner complex can be considered to consist of three layers: the iliotibial tract and the superficial portion of the biceps femoris form the first layer, the LCL the second layer, and the joint capsule, the arcuate ligament, the popliteofibular ligament, and the popliteal tendon constitute the third layer. The LCL is the primary static stabiliser to the lateral opening of the joint supplemented by the popliteofibular ligament and the cruciates. The popliteofibular ligament is the primary restraint to posterolateral rotation, supplemented by the LCL and the popliteus tendon. ${ }^{16}$

The following tests are most useful in differentiating between isolated LCL, PCL, and PLC or combined PCLPLC injuries. Care must be taken to ensure that there is no neurovascular injury in particular to the common peroneal nerve.

(1) Varus stress test. This is performed at full extension and at $15^{\circ}$ of flexion. Increased lateral opening at $15^{\circ}$ of flexion indicates LCL and possibly PLC injury. Slightly increased lateral opening even at full extension is consistent with combined injury to the LCL and PLC. Significant opening at full extension indicates additional injury to the PCL and possibly the ACL. Comparison with the normal side is important. 


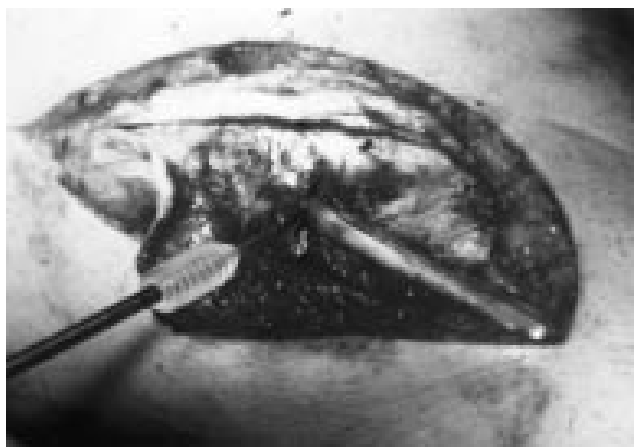

Figure 2 Posterolateral reconstruction using hamstring tendons secured by an absorbable interference screw.

(2) Passive external rotation of the tibia (relative to the femur) with the knee at $30^{\circ}$ and $90^{\circ}$ of flexion. This is best performed with the patient prone. In the rare case of isolated posterolateral injury, increased external rotation is noted at $30^{\circ}$ but not at $90^{\circ}$. When combined PCL and PLC injuries are present, increased external rotation is noted in both positions. External rotation of the injured knee of $10^{\circ}$ or more compared with the normal knee is considered significant. In addition, the tibial condyles are palpated to determine their position relative to the femur to ensure that the increased external rotation is from posterolateral rotary instability and not anteromedial instability.

Tests such as the external rotation recurvatum test, reversed pivot shift test, and a posterior drawer test performed with the foot in external rotation - that is, the posterolateral drawer test-may also be performed for additional confirmation but are not particularly specific.

Limb alignment and gait pattern must be observed to ensure there is no lateral thrust on walking. If this is not recognised, the ligament reconstruction may fail in the absence of a corrective osteotomy. MR scanning is useful in the acute setting not only to identify associated cruciate injury but also to help plan surgery by identifying the site of injury to the structures in the PLC.

MANAGEMENT OF LCL AND PLC INJURIES

The data available on surgical outcomes for posterolateral reconstruction are limited. The wide variety of procedures used to treat patients with posterolateral instability makes it difficult to derive a consensus on the most effective and appropriate approach to this clinical entity. With acute injuries of the PLC, surgical intervention within two weeks of the initial injury is optimal ${ }^{17}$ with the direct repair of all injured structures where possible. If the LCL or the popliteofibular ligament is ruptured mid substance, then consideration should be given to reconstruction of these structures, as direct repair in isolation may be insufficient. In the chronic setting, direct repair is rarely possible and a variety of techniques can be used, including tissue advancement and augmentation with autograft or allograft tissues. We use hamstring tendon autografts to reconstruct the LCL and popliteofibular ligament similar to the technique of Larson as described by Kumar et $a l^{18}$ (fig 2). If there is a varus thrust, we prefer to perform an opening medial wedge osteotomy to avoid any further slackening of the lateral structures that may be seen with a lateral closing wedge osteotomy (fig 3).

\section{Posterior cruciate ligament}

ANATOMY AND FUNCTION

PCL injuries account for $15-20 \%$ of knee ligament injuries ${ }^{19}$ and they are increasingly being recognised. The PCL originates from the medial femoral condyle, with its

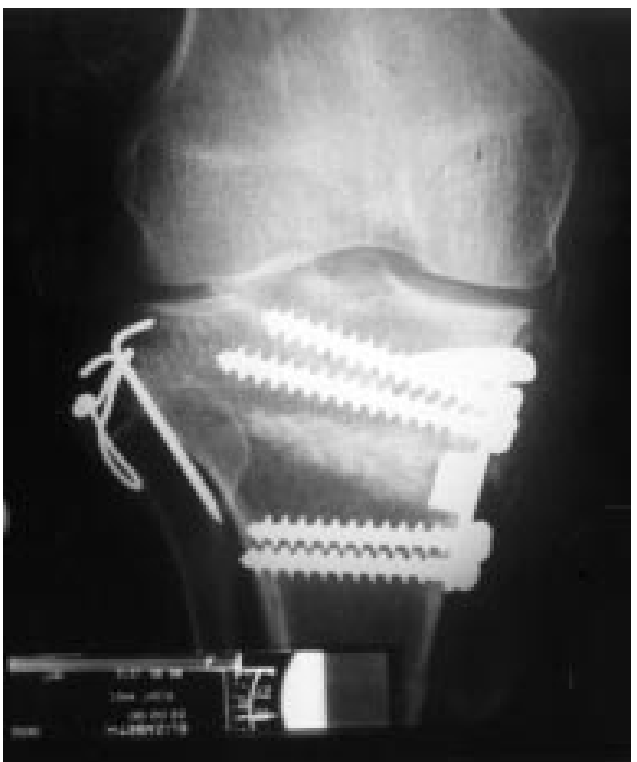

Figure 3 Anteroposterior radiograph of the knee showing fixation of a medial opening wedge osteotomy. There has been a previous fixation for traumatic avulsion of the fibula head.

attachment in the shape of a semicircle. It inserts into a depression between the posterior aspect of the two tibial plateaux, about $1 \mathrm{~cm}$ below the articular surface. Functionally it is composed of two bundles, anterolateral and posteromedial. In mid range of flexion $\left(40-120^{\circ}\right)$, the anterolateral bundle is the primary restraint to posterior drawer. The posteromedial bundle increases its contribution towards full flexion. ${ }^{20}$ Previous studies have shown the anterolateral bundle to be structurally and biomechanically more important. ${ }^{21}$ Recent studies ${ }^{22}$ recommend reconstruction of both the bundles to restore function of the PCL throughout the range of motion.

PCL is the primary static restraint to posterior translation of the tibia. It is a secondary stabiliser to varus angulation and external tibial rotary displacement at $90^{\circ}$ of knee flexion. The mechanism of most sporting PCL injuries is a fall on the flexed knee. This imparts a backward force to the tibial tubercle which ruptures the ligament, usually in isolation. Hyperflexion of the knee without a direct blow to the tibia can also cause isolated PCL injury. Forced hyperextension can injure the PCL, but this is usually combined with injury to the ACL. Posteriorly directed force to the anteromedial tibia with the knee in hyperextension may also cause a PLC injury. Significant varus or valgus stress will injure the PCL only after rupture of the appropriate collateral ligament. In isolation, there is often little instability, whereas, when associated with posterolateral or posteromedial injuries, stability of the knee is dramatically reduced.

We consider the following tests to be the most useful for diagnosing isolated PCL injury.

(1) The posterior sag test. This is performed at $90^{\circ}$ of hip and knee flexion and uses gravity to apply a posteriorly directed force to the tibia. Posterior displacement of the tibia indicates PCL injury.

(2) The posterior drawer test at $90^{\circ}$ of flexion. This test is performed with the patient supine with both feet on the table and the knees flexed to $90^{\circ}$. At this angle of flexion the anterior tibial condyles should be anterior to the corresponding femoral condyles. The injured knee is compared with the normal knee and the posterior translation is measured as grade $\mathrm{I}$ if it is $0-5 \mathrm{~mm}$ (tibia still anterior to femur), grade II if $5-10 \mathrm{~mm}$ (tibia flush with femur) and grade III if over $10 \mathrm{~mm}$ with no 
Table 1 Clinical tests in posterior cruciate ligament (PCL)/posterolateral corner (PLC) instability

\begin{tabular}{llll}
\hline & PLC & PLC + PCL & \multirow{2}{*}{$P C L$} \\
\hline Posterior sag at $90^{\circ}$ & $\mathrm{N}$ & $\mathrm{SP}$ & $\mathrm{SP}$ \\
Posterior drawer at $90^{\circ}$ & $\mathrm{N}$ & $\mathrm{SP}$ & $\mathrm{SP}$ \\
Quadriceps active test & $\mathrm{N}$ & $\mathrm{SP}$ & $\mathrm{SP}$ \\
Passive external rotation at $30^{\circ}$ & $\mathrm{P}$ & $\mathrm{SP}$ & $\mathrm{N}$ \\
Passive external rotation at $90^{\circ}$ & $\mathrm{P}$ & $\mathrm{SP}$ & $\mathrm{SP}$
\end{tabular}

$\mathrm{N}$, Negative; P, positive; SP, strongly positive.

end point (tibial condyles sagging behind femoral condyles). In the PCL deficient knee, the Lachman test may show increased anteroposterior translation but a firm anterior end point. The increased anteroposterior translation here is due to the posteriorly subluxed tibia being reduced into its normal position.

(3) The quadriceps active test. The patient is supine, knees flexed to $90^{\circ}$ with the foot resting on the table. Anterior translation of the proximal tibia with quadriceps contraction indicates PCL injury.

Tests for posterolateral rotary instability as described above should also be performed to detect combined injuries (table 1).

Plain radiographs may show a PCL avulsion fracture. MRI has proved to be sensitive and specific for diagnosis of acute PCL injury and it can detect meniscal and chondral damage.

\section{MANAGEMENT OF INJURIES}

\section{Acute isolated PCL injuries}

Reconstruction is usually not required for the treatment of isolated acute PCL injuries. ${ }^{2}$ If the degree of posterior translation is less than $10 \mathrm{~mm}$ as in most isolated injuries or even in those with small tibial PCL avulsion fractures, a non-operative aggressive rehabilitation programme is arranged. After an initial period of RICE, an extension splint is worn for three or four weeks. Physiotherapy is focused especially on quadriceps strengthening. Close follow up is necessary to avoid combined instability being missed. If an avulsed fragment is large, it can be reduced and fixed through a posterior approach. If the posterior translation is greater than $10 \mathrm{~mm}$ without a firm end point - that is, grade 3-reconstruction is advised because it is likely that additional secondary restraints have been compromised. ${ }^{23}$ If significant chondral or meniscal injuries are suspected on the basis of MRI, an arthroscopy is performed to deal with them.

Acute surgical treatment of complete PCL tears can include primary repair or reconstruction depending on the location of injury. PCL reconstruction can be performed with a patellar tendon autograft, semitendinosus and gracilis autograft, or a patella or Achilles tendon allograft, and can be by open or arthroscopically assisted techniques. The arthroscopic procedure is performed with radiographic assistance (fig 4) using an additional posteromedial

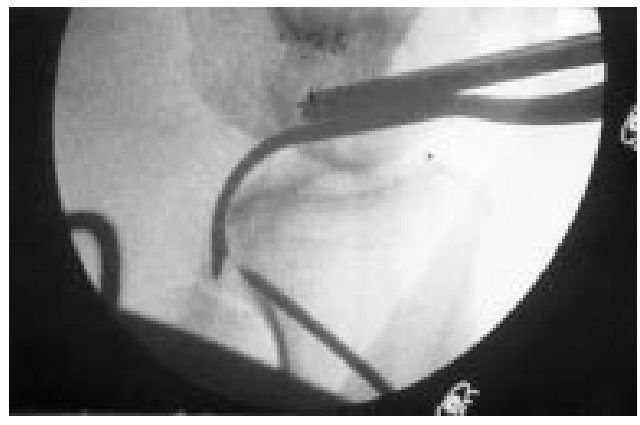

Figure 4 Intraoperative radiograph to ensure correct positioning of the tibial tunnel during reconstruction of the posterior cruciate ligament. portal to assist in tibial tunnel preparation. This procedure is technically demanding.

\section{Acute combined injuries}

With combined PLC, ACL or grade III MCL injury which may occur in a spontaneously reduced dislocation of the knee, it appears best to operate early, between two and three weeks, to maximise healing potential and minimise stiffness. ${ }^{24}$ One must particularly watch for any neurovascular injury.

\section{Chronic isolated PCL instability}

Parolie and Bergfeld ${ }^{25}$ reported long term results of non-operative treatment of isolated PCL injuries. At an average follow up of 6.2 years, $80 \%$ of the patients were satisfied with their results and $84 \%$ had returned to their previous sport. Rehabilitation of the quadriceps to achieve the same strength as on the non-injured side correlated with a successful result. Whether the PCL deficient knee is at risk of developing degenerative changes is not clear. Despite the lack of prospective studies, it appears that progressive degenerative changes may occur in some PCL deficient knees. Long term results of surgical reconstructions for PCL instability remain unclear.

We recommend non-operative treatment with quadriceps rehabilitation for most patients initially. Reconstruction is considered if the laxity is more than $10 \mathrm{~mm}$ at $90^{\circ}$ of knee flexion or in the presence of symptoms that have not responded to rehabilitation treatment. Reconstruction is not performed if there is evidence of considerable degenerative change. No data are available to support reconstruction for pain. We use hamstring autograft for the reconstruction, reconstituting both bundles of the PCL with a suspensory form of fixation on the tibial side and interference fixation with bioabsorbable screws on the femoral side.

\section{Chronic combined injuries}

This is a difficult problem. The principles of management include proper evaluation of the associated structures involved and correction of limb alignment for reasons alluded to earlier.

\section{REHABILITATION}

Rehabilitation after PCL reconstruction is designed to restore range of motion without stressing the graft. Exercises that produce posterior tibial translation are avoided. Limited weight bearing using crutches is allowed with a PCL knee brace allowing full range of movements for six weeks. After the early rehabilitation programme, running begins at about five months, and sports and physical activity at six to seven months. A full range of sport is allowed when adequate quadriceps and hamstring strength is shown after about nine months.

\section{The future}

It is hoped that future basic science and clinical studies and further technical experience with various reconstructive procedures will continue to improve both our understanding and surgical outcomes for instability of the knee. Collagen engineering and developments in prosthetic ligaments are likely to play an increasing role, avoiding graft harvest. Long term studies on the outcomes of PCL reconstruction may clarify whether early reconstruction of the PCL prevents late degenerative changes. Long term studies to evaluate the role of factors other than mechanical, such as local intra-articular cytokines released at the time of injury, in the development of degenerative changes 
in chronic ligament injury are being explored. ${ }^{26}$ This could lead to prevention of early degenerative changes in articular cartilage after ligament injuries.

Specialist Registrar in Orthopaedic Surgery

T K KAKARLAPUDI

The Northern General Hospital

Sheffield S5 $7 \mathrm{AU}$

Consultant Orthopaedic Surgeon

D R BICKERSTAFF

Thornbury Hospital

Sheffield S10 3BR

United Kingdom

email:drbickerstaff@uk-consultants

1 Indelicato PA. Isolated MCL injuries in the knee. $\mathcal{F}$ Am Acad Orthop Surg 1995;3:9-14.

2 Shahane SA, Bickerstaff DR. Proximal advancement of the medial collateral ligament for chronic medial instability of the knee joint. The Knee 1998;5: 191-7.

3 Amis AA et al. Functional anatomy of ACL: fibre bundle action related to ligament replacement and injuries. F Bone foint Surg [Br] 1991;73:260-7.

4 Biedert RM, Stauffer E, Friederich NF. Occurence of free nerve endings in the soft tissues of the knee joint: a histologic investigation. Am 7 Sports Med 1992;20:430-3.

5 Fu FH, Harner CD, Johnson DL, et al. Biomechanics of knee ligaments: basic concepts and clinical applications. I Bone foint Surg [Am]

6 Glashow JL et al. Double blind assessment of the value of MRI in the diagnosis of ACL meniscal lesions. F Bone foint Surg [Am] 1989;71;113-19.

7 Graf BK, Cook DA, De Smet AA, et al. Bone bruises on MRI evaluation of Graf BK, Cook DA, De Smet AA, et al. Bone br
ACL injuries. Am $\mathcal{f}$ Sports Med 1993;22:220-3.

8 Harner CD, Irrgang JJ, Paul J, et al. Loss of motion after ACL reconstruction. Am $\mathcal{f}$ Sports Med 1992;20:499-506.

9 Frank CB, Jackson DW. The science of reconstruction of ACL. $\mathcal{F}$ Bone foint Surg $[\mathrm{Am}]$ 1997;79:1556-76.
10 Daniel DM, Stone ML, Dobson BE, et al. Fate of the ACL injured patient: a prospective outcome study. Am F Sports Med 1994;22:632-44.

11 Hanks GA, Gause TM, Handal JA et al. Meniscus repair in the ACL deficient knee. Am f Sports Med 1990;18:606-13.

12 Wojtys EM, Huston LJ. Functional knee braces: the 25 year controversy. In: Chan KM, Fu F, Maffulli N, eds. Controversies in orthopaedic sports medicine. Hong Kong: Williams \& Wilkins, 1998:106-15.

13 Yasuda K, Tsujimo J, et al. Graft site morbidity with autogenous semitendinosus/gracilis tendons. Am F Sports Med 1995;23:206-14.

14 Otero AL, Hutcheson L. A comparison of the doubled semitendinosus/ gracilis and central third of the patellar tendon autografts in arthroscopic ACL reconstruction. Arthroscopy 1993;9:143-8.

15 Shelbourne KD, Nitz P. Accelerated rehab after ACL reconstruction. Am $\mathcal{f}$ Sports Med 1990;18:292-9.

16 Shahane SA, Ibbotson C, Bickerstaff DR, et al. The popliteo fibular ligament: an anatomic study of the posterolateral corner of the knee. $\mathcal{F}$ Bone foint Surg [Br] 1999;81:636-42.

17 Jakob RP, Middleton RG. Postero lateral instability. In: Chan KM, Fu F, Maffulli N, eds. Controversies in orthopaedic sports medicine. Hong Kong: Maffulli N, eds. Controversies in orthopaedic sports medicine. Hong Kong:

18 Kumar A, Jones S, Bickerstaff DR. Posterolateral reconstruction of the knee: a tunnel technique for proximal fixation. The Knee 1999;6:257-60.

19 Cooper DE, Warren RF, et al. The PCL and the posterolateral structures of the knee: anatomy, function and patterns of injury. Instr Course Lect 1991: 40:249-70.

20 Race A, Amis AA. Loading of the two bundles of the PCL: an analysis of bundle function in a posterior drawer. F Biomech 1996;29:873-9.

21 Harner CD, Xerogeanes JW. The human PCL complex: an interdisciplinary study: ligament morphology and biomechanical events. Am 7 Sports Med 1995;23:736-45.

22 Race A, Amis AA, et al. PCL reconstruction: In vitro biomechanical comparison of "isometric" versus single and double bundle anatomic grafts. F Bone foint Surg [Br] 1998;80:173-9.

23 Veltri DM, Warren RF. Isolated and combined PCL injuries. F Am Acad Orthop Surg 1993;1:67-75.

24 Good L, Johnson RJ. The dislocated knee. fAAOS 1995;3:284-92.

25 Parolie JM, Bergfeld JA. Long term results of non operative treatment of isolated PCL injuries in the athlete. Am $\mathcal{F}$ Sports Med 1986;14:35-8.

26 Price JS, Till SH, Bickerstaff DR, et al. Degradation of cartilage type II collagen precedes the onset of OA following ACL rupture. Arthritis Rheum $1999 ; 42: 2390-8$ 